\title{
ON TWO THEOREMS CONCERNING SETS WITH CONVEX SECTIONS
}

\author{
E. Tarafdar
}

In this note two theorems of Ma and Fan concerning sets with convex sections are generalised.

In this note we generalise two theorems of Fan concerning sets with convex sections. These generalisations involve a relaxation of the compactness conditions on the family of sets. Fan ([4], Theorem 16) proved Theorem I which includes the theorem of $\mathrm{Ma}$ [5]. These results are usually proved by using fixed point theorems or the KnasterKuratowski-Mazurkiewicz theorem.

The following notation is used throughout.

Let $\left\{X_{a}: \alpha \in I\right\}$ be a family of nonempty sets, where $I$ is an index set. We denote the cartesian product by $X$, that is $X=\prod_{\alpha \in I} X_{\alpha}$ and for each $\alpha \in I, \hat{X}_{\alpha}=\prod_{\substack{\beta \in I \\ \alpha \neq \beta}} X_{\beta}$. Also $\hat{x}_{\alpha}$ denotes an element of $\hat{X}_{\alpha}$. For a subset $A$ of $X$, for each $\alpha \in I$ and each $x_{\alpha} \in X_{\alpha}$, let $A\left(x_{\alpha}\right)=\left\{\hat{x}_{\alpha} \in \hat{X}_{\alpha}:\left[x_{\alpha}, \hat{x}_{\alpha}\right] \in A\right\}$ and for each $\alpha \in I$ and each $\hat{x}_{\alpha} \in \hat{X}_{\alpha}$, let $A\left(\hat{x}_{\alpha}\right)=\left\{x_{\alpha} \in X_{\alpha}:\left[x_{\alpha}, \hat{x}_{\alpha}\right] \in A\right\}$. Also note for each $\alpha \in I, X_{\alpha} \times \hat{X}_{\alpha}=X$ (with the appropriate ordering).

ThEOREM 1. Suppose each $X_{\alpha}$ is a compact convex subset of a Hausdorff topological vector space $E_{\alpha}$

Let $\left\{A_{\alpha}: \alpha \in I\right\}$ and $\left\{B_{\alpha}: \alpha \in I\right\}$ be two families of subsets of $X$ having the following properties:

(a) for each $\alpha \in I$ and each $x_{\alpha} \in X_{\alpha}$, the set $B_{\alpha}\left(x_{\alpha}\right)$ is open in $\hat{X}_{\alpha}$;

(b) for each $\alpha \in I$ and each $\hat{x}_{\alpha} \in \hat{X}_{\alpha}$, the set $B_{\alpha}\left(\hat{x}_{\alpha}\right)$ in non-empty and the set $A_{\alpha}\left(\hat{x}_{\alpha}\right)$ contains the convex hull of $B_{\alpha}\left(\hat{x}_{\alpha}\right)$.

Then $\bigcap_{\alpha \in I} A_{\alpha} \neq \emptyset$.

We use this theorem to prove the following extended version in which we remove the compactness condition on each $X_{\alpha}$ and add condition (c) below. Condition (c) has been used by Fan ([4], Theorem 15) in the case of a single finite family.

Received 4 February 1988

Copyright Clearance Centre, Inc. Serial-fee code: 0004-9729/88 \$A2.00+0.00. 
THEOREM 2. Suppose each $X_{\alpha}$ is a convex subset of a Hausdorff topological vector space $E_{\alpha}$.

Let $\left\{A_{\alpha}: \alpha \in I\right\}$ and $\left\{B_{\alpha}: \alpha \in I\right\}$ be two families of subsets of $X$ satisfying properties (a) and (b) above. Further assume that

(c) there is a non-empty compact convex subset $K$ of $X$ such that for every $x \in X \backslash K, x=\left[x_{\alpha}, \hat{x}_{\alpha}\right] \in X_{\alpha} \times \hat{X}_{\alpha}$, there exists $y \in K, y=\left[y_{\alpha}, \hat{y}_{\alpha}\right] \in$ $X_{\alpha} \times \hat{X}_{\alpha}$ satisfying $\left[y_{\alpha}, \hat{x}_{\alpha}\right] \in B_{\alpha}$ for each $\alpha \in I$.

Then there exists at least one point $x_{0}$ such that $X_{0} \in \underset{\alpha \in I}{\cap} A_{\alpha}$.

Proof: Let $P_{\alpha}: X \rightarrow X_{\alpha}$ be the projection of $X$ onto $X_{\alpha}$ for each $\alpha \in I$. For each $\alpha \in I$, let $P_{\alpha}(K)=X_{\alpha}^{\prime}$, which is non-empty compact and convex. Let $X^{\prime}=\prod_{\alpha \in I} X_{\alpha}^{\prime}$. For each $\alpha \in I$, we let $A_{\alpha}^{\prime}=A_{\alpha} \cap X^{\prime}$ and $B_{\alpha}^{\prime}=B_{\alpha} \cap X^{\prime}$. We also set $\hat{X}_{\alpha}^{\prime}=\prod_{\substack{\beta \in I \\ \beta \neq \alpha}} X_{\beta}^{\prime}$.

Then for each $\alpha \in I$ and each $x_{\alpha} \in X_{\alpha}^{\prime}$, it is easily seen that the set

$$
B_{\alpha}^{\prime}\left(x_{\alpha}\right)=\left\{\hat{x}_{\alpha} \in \hat{X}_{\alpha}^{\prime}:\left[x_{\alpha}, \hat{x}_{\alpha}\right] \in B_{\alpha}^{\prime}\right\}=\left\{\hat{x}_{\alpha} \in \hat{X}_{\alpha}:\left[x_{\alpha}, \hat{x}_{\alpha}\right] \in B_{\alpha}\right\} \cap \hat{X}_{\alpha}^{\prime}
$$

which is relatively open in $\hat{X}_{\alpha}^{\prime}$ by condition (a).

Also for each $\alpha \in I$ and each $\hat{x}_{\alpha} \in \hat{X}_{\alpha}^{\prime}$, the set

$$
B_{\alpha}^{\prime}\left(\hat{x}_{\alpha}\right)=\left\{x_{\alpha} \in \hat{X}_{\alpha}^{\prime}:\left[x_{\alpha}, \hat{x}_{\alpha}\right] \in B_{\alpha}^{\prime}\right\}
$$

is non-empty for the following reasons. By condition (b) $B_{\alpha}\left(\hat{x}_{\alpha}\right) \neq \emptyset$. Let $x_{\alpha} \in$ $B_{\alpha}\left(\hat{x}_{\alpha}\right)$. If $x_{\alpha} \notin B_{\alpha}^{\prime}\left(\hat{x}_{\alpha}\right)$, that is $x=\left[x_{\alpha}, \hat{x}_{\alpha}\right] \notin B_{\alpha}^{\prime}=B_{\alpha} \cap X^{\prime}$. Then since $x=\left[x_{\alpha}, \hat{x}_{\alpha}\right] \in B_{\alpha}$, it follows that $x \notin X^{\prime}$ and hence $x \notin K$ as $K \subset X^{\prime}$. By condition (c) there exists $y \in K, y=\left[y_{\alpha}, \hat{y}_{\alpha}\right] \in X_{\alpha} \times \hat{X}_{\alpha}$ such that $\left[y_{\alpha}, \hat{x}_{\alpha}\right] \in B_{\alpha}$ for each $\alpha \in I$. Thus as $y_{\alpha} \in P_{\alpha}(K)=X_{\alpha}^{\prime}$ and $\hat{x}_{\alpha} \in \hat{X}_{\alpha}^{\prime},\left[y_{\alpha}, \hat{x}_{\alpha}\right] \in B_{\alpha} \cap X^{\prime}=B_{\alpha}^{\prime}$. Hence $y_{\alpha} \in B_{\alpha}^{\prime}\left(\hat{x}_{\alpha}\right)$. Therefore $B_{\alpha}^{\prime}\left(\hat{x}_{\alpha}\right)$ is always non-empty.

Finally it can be easily seen that for each $\alpha \in I$ and $\hat{x}_{\alpha} \in \hat{X}_{\alpha}^{\prime}$,

$$
A_{\alpha}^{\prime}\left(\hat{x}_{\alpha}\right)=\left\{x_{\alpha} \in X_{\alpha}^{\prime}:\left[x_{\alpha}, \hat{x}_{\alpha}\right] \in A_{\alpha}^{\prime}\right\}=\left\{x_{\alpha} \in X_{\alpha}:\left[x_{\alpha}, \hat{x}_{\alpha}\right] \in A_{\alpha}\right\} \cap X_{\alpha}^{\prime}
$$

contains the convex hull of the set $B_{\alpha}^{\prime}\left(\hat{x}_{\alpha}\right)=\left\{x_{\alpha} \in X_{\alpha}^{\prime}:\left[x_{\alpha}, x_{\alpha}^{\prime}\right] \in B_{\alpha}^{\prime}\right\}=\left\{x_{\alpha} \in\right.$ $\left.X_{\alpha}:\left[x_{\alpha}, \hat{x}_{\alpha}\right] \in B_{\alpha}\right\} \cap X_{\alpha}^{\prime}$ by condition (c). Hence the system $\left\{X_{\alpha}^{\prime}, A_{\alpha}^{\prime}, B_{\alpha}^{\prime}\right\}$ satisfies the conditions of Theorem 1 .

Therefore $\underset{a \in I}{\cap} A_{\alpha}^{\prime} \neq \emptyset$. Hence $\underset{\alpha \in I}{\cap} A_{\alpha} \neq 0$. 
Remark. Theorem 2 for a finite family has been obtained in [9] as an application of the fixed theorem proved in [7]. The technique applied in [9] fails for the case of an arbitrary family as the product of an arbitrary family of open sets need not be open.

The following theorem, which includes a theorem of Von Neumann [6], which in turn implies the fundamental theorem of two-person zero sum games was proved by Fan ([2] and [3]) and also Browder [1].

THEOREM 3. Suppose each $X_{\alpha}$ is a compact convex subset of a locally convex Hausdorff topological vector space $E_{\alpha}$. Let $\left\{A_{\alpha}: \alpha \in I\right\}$ be a family of closed subsets of $X$ having the property: for each $\alpha \in I$ and each $\hat{x}_{\alpha} \in \hat{X}_{\alpha}$, the set $A\left(\hat{x}_{\alpha}\right)$ is a non-empty convex set.

Then $\bigcap_{\alpha \in I} A_{\alpha} \neq \emptyset$.

Using this theorem we prove the following extension.

Theorem 4. Suppose each $X_{\alpha}$ is a convex subset of a locally convex Hausdorff topological vector space $E_{\alpha}$. Let $\left\{A_{\alpha}: \alpha \in I\right\}$ be a family of closed subsets of $X$ having the property: for each $\alpha \in I$ and each $\hat{x}_{\alpha} \in \hat{X}_{\alpha}$ the set $A_{\alpha}\left(\hat{x}_{\alpha}\right)$ is a non-empty convex set.

Further assume that there is a non-empty compact convex subset $K$ of $X$ such that for every $x \in X \backslash K, x=\left[x_{\alpha}, \hat{x}_{\alpha}\right] \in X_{\alpha} \times \hat{x}_{\alpha}$, there exists $y \in K, y=\left[y_{\alpha}, \hat{y}_{\alpha}\right] \in$ $X_{\alpha} \times \hat{X}_{\alpha}$ satisfying $\left[y_{\alpha}, \hat{x}_{\alpha}\right] \in A_{\alpha}$ for each $\alpha \in I$.

Then there exists at least one point $x_{0}$ such that $x_{0} \in \underset{\alpha \in I}{\cap} A_{\alpha}$.

Proof: As before, let $P_{\alpha}: X \rightarrow X_{\alpha}$ be the projection of $X$ onto $X_{\alpha}$ for each $\alpha$. For each $\alpha \in I$, let $P_{\alpha}(K)=X_{\alpha}^{\prime}$ which is a non-empty compact convex set in $E_{\alpha}$. Let $X^{\prime}=\prod_{\alpha \in I} X_{\alpha}^{\prime}$ and for each $\alpha \in I$, let $A_{\alpha}^{\prime}=A_{\alpha} \cap X^{\prime}$. Then $A_{\alpha}^{\prime}$ is a closed subset of $X$ for each $\alpha \in I$ as $X^{\prime}$ is compact and $A_{\alpha}$ is closed.

Now for each $\alpha \in I$ and each $\hat{x}_{\alpha} \in \hat{X}_{\alpha}$ we can prove by giving similar arguments to those in the proof of Theorem 2 that $A_{\alpha}^{\prime}\left(\hat{x}_{\alpha}\right)=\left\{x_{\alpha} \in X_{\alpha}^{\prime}:\left[x_{\alpha}, \hat{x}_{\alpha}\right] \in A_{\alpha}^{\prime}\right\} \neq \emptyset$. That $A^{\prime}\left(\hat{x}_{\alpha}\right)$ is convex follows from the equality:

$$
A_{\alpha}^{\prime}\left(\hat{x}_{\alpha}\right)=\left\{x_{\alpha} \in X_{\alpha}^{\prime}:\left[x_{\alpha}, \hat{x}_{\alpha}\right] \in A_{\alpha}^{\prime}\right\}=\left\{x_{\alpha} \in X_{\alpha}:\left[x_{\alpha}: \hat{x}_{\alpha}\right] \in A_{\alpha}\right\} \cap X_{\alpha}^{\prime}
$$

Now applying Theorem 3 to the system $\left\{X_{\alpha}^{\prime}, A_{\alpha}^{\prime}\right\}$ we have $\underset{\alpha \in I}{\cap} A_{\alpha}^{\prime} \neq 0$.

Thus the theorem is proved.

Remark. Results dual, in the sense of $[8]$, to those of Theorems 2 and 4 can be obtained similarly. 


\section{References}

[1] F.E. Browder, 'Fixed point theory of multivalued mappings in topological vector spaces', Math. Ann. 177 (1968), 283-301.

[2] K. Fan, 'Fixed-point and minimax theorems in locally convex topological linear spaces', Proc. Nat. Acad. Sci U.S.A. 38 (1952), 121-126.

[3] K. Fan, 'Applications of a theorem concerning sets with convex sections', Math. Ann. 163 (1966), 189-203.

[4] K. Fan, 'Some properties of convex sets related to fixed point theorems', Math. Ann. 266 (1984), 519-537.

[5] T.W. Ma, 'On sets with convex sections', J. Math. Anal. Appl. 27 (1969), 413-416.

[6] J. Von Neumann, 'Zur Theorie der Gesellschaftsspiele', Math. Ann. 100 (1928), 295-320.

[7] E. Tarafdar, 'A fixed point theorem equivalent to Fan-Knaster-Kuratowski-Mazurkiewicz's theorem', J. Math. Anal. Appl. 128 (1987), 475-479.

[8] E. Tarafdar and T. Husain, 'Duality in fixed point theory of multivalued mappings with application', J. Math. Anal. Appl. 63 (1978), 373-376.

[9] E. Tarafdar, 'A theorem concerning sets with convex sections', Ramanujan Centary Volume, Indian J. Math. (to appear).

Department of Mathematics

University of Queensland

St. Lucia, 4067

Brisbane, Australia 